Red-Shift

\title{
(2-Hydroxyphenyl)-Benzothiazole Emission by Mimicking the Excited-State Intramolecular Proton Transfer Effect
}

\author{
Yong Ren ${ }^{1 \dagger}$, Lei Zhou ${ }^{1,2+}$ and Xin $\mathrm{Li}^{1 *}$ \\ ${ }^{1}$ College of Pharmaceutical Sciences, Zhejiang University, Hangzhou, China, ${ }^{2}$ Collaborative Innovation Center of Yangtze River \\ Delta Region Green Pharmaceuticals, Zhejiang University of Technology, Hangzhou, China
}

OPEN ACCESS

Edited by:

Yao Sun,

Central China Normal University,

China

Reviewed by:

Youjun Yang,

East China University of Science and

Technology, China

Zhaochao Xu,

Dalian Institute of Chemical Physics

(CAS), China

Lin Li,

Northwestern Polytechnical

University, China

*Correspondence:

Xin $\mathrm{Li}$

lixin81@zju.edu.cn

${ }^{+}$These authors have contributed equally to this work

Specialty section:

This article was submitted to

Organic Chemistry,

a section of the journal

Frontiers in Chemistry

Received: 02 November 2021

Accepted: 06 December 2021

Published: 24 December 2021

Citation:

Ren Y, Zhou L and Li X (2021) RedShift (2-Hydroxyphenyl)-Benzothiazole Emission by Mimicking the Excited-

State Intramolecular Proton

Transfer Effect.

Front. Chem. 9:807433.

doi: $10.3389 /$ fchem.2021.807433
Novel strategies to optimize the photophysical properties of organic fluorophores are of great significance to the design of imaging probes to interrogate biology. While the 2-(2hydroxyphenyl)-benzothiazole (HBT) fluorophore has attracted considerable attention in the field of fluorescence imaging, its short emission in the blue region and low quantum yield restrict its wide application. Herein, by mimicking the excited-state intramolecular proton transfer (ESIPT) effect, we designed a series of 2-(2-hydroxyphenyl)-benzothiazole (HBT) derivatives by complexing the heteroatoms therein with a boron atom to enhance the chance of the tautomerized keto-like resonance form. This strategy significantly red-shifted the emission wavelengths of HBT, greatly enhanced its quantum yields, and caused little effect on molecular size. Typically, compounds 12B and 13B were observed to emit in the near-infrared region, making them among the smallest organic structures with emission above $650 \mathrm{~nm}$.

Keywords: fluorescence imaging, fluorophore, benzothiazole, excited-state intramolecular proton transfer, tautomerization, wavelength, quantum yield

\section{INTRODUCTION}

Novel design of new fluorophores has attracted increasing attention due to their fascinating applications in fields such as fluorescence imaging (Kim and Cho 2015; Fernández and Vendrell, 2016; Singh et al., 2019; Tian et al., 2021), fluorescence diagnosis (Vahrmeijer et al., 2013; Goryaynov et al., 2019; Hernot et al., 2019), or as light-emitting diodes (LEDs) (Yang et al., 2017; Liu et al., 2018). In particular, organic small-molecule fluorophores are extremely popular due to their well-defined structures, easy synthesis, and consistency between batches. For an organic small-molecule fluorophore, its chemical structure determines its fluorescence properties including fluorescence excitation wavelength, emission wavelength, Stokes shift and quantum yield. And these fluorescence properties greatly determine the application scope of the fluorophore in various research fields. Under this circumstance, the design of new small-molecule fluorophores with desirable properties has attracted much attention (Yuan et al., 2012; Cheng et al., 2016; Qian et al., 2017; Xiang et al., 2019). Generally, there are two strategies widely used for new fluorophore development; one is de novo design (Xiang et al., 2019), and the other is structure optimization of classical fluorophores such as BODIPY (Ulrich et al., 2008; Boens et al., 2012; Lu et al., 2014; Wang et al., 2020), rhodamine (Lefevre et al., 1996; Fu et al., 2008; Song et al., 2008; Koide et al., 2012; Grimm et al., 2015; Chu et al., 2016; Liu et al., 2016a; Grimm et al., 2017; Lei et al., 2017; Grimm et al., 2020), cyanine (Michie et al., 2017; Usama et al., 2019; Li et al., 2020a). The latter strategy is especially appealing because the classical 


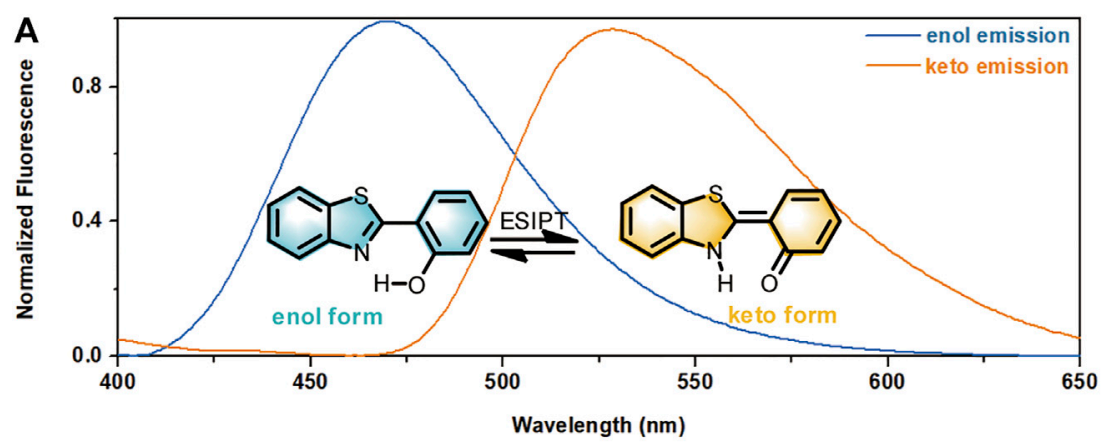

B

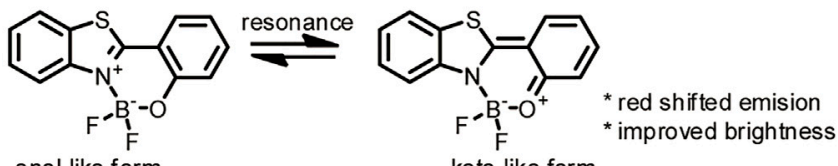

C

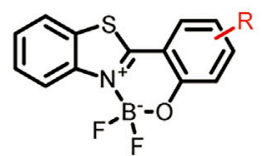

$1 \mathrm{~B}$, meta-, $\mathrm{R}=\mathrm{NO}_{2}$
$2 \mathrm{~B}$, meta-, $\mathrm{R}=\mathrm{Br}$
$3 \mathrm{~B}$, meta-, $\mathrm{R}=\mathrm{F}$
$4 \mathrm{~B}$, meat,$- \mathrm{R}=\mathrm{OMe}$
$5 \mathrm{~B}$, meta-, $\mathrm{R}=\mathrm{NH}_{2}$

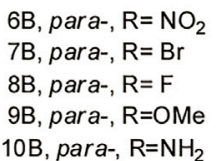

FIGURE 1 | Design rationale for $\mathrm{HBT}_{-} \mathrm{BF}_{2}$ fluorophores. (A) Enol and keto forms of HBT structures and their emissions. (B) The HBT-BF ${ }_{2}$ structure and its resonance to a keto-like structure. (C) Structures of $\mathrm{HBT}^{-\mathrm{BF}_{2}}$ series of fluorophores with various substituents.

fluorophores usually show good photophysical properties which set a good starting point for further structure modification.

2-(2-Hydroxyphenyl)-benzothiazole (HBT) is a wellrecognized fluorophore with unique properties such as good photostability and solid-based emission. This fluorophore has attracted dramatic interest in the field of bio-imaging (Li et al., 2015; Barman et al., 2016; Li et al., 2020b). However, there are several disadvantages regarding this fluorophore, e.g., its short emission wavelength and low quantum yield. The development of HBT derivatives with longer emission wavelength and improved quantum yields is highly desirable. Herein, we reported the design and synthesis of a new series of HBT derivatives, among which, compounds 12B and 13B demonstrated not only near-infrared emission, but significantly improved brightness compared to HBT. Their applicability for live cell imaging was also confirmed. These improved features demonstrate the feasibility of our design strategy, and potentiate the wider application scope for $\mathrm{HBT}$ and its derivatives.

\section{RESULTS}

\section{Design and Synthesis of 2-(2-Hydroxyphenyl)-Benzothiazole Derivatives Inspired by Excited-State Intramolecular Proton Transfer Effect}

Traditionally, extending the conjugation system is a wellrecognized strategy to red-shift compound absorption and emission. This is exemplified by the development of the Cyanine family fluorophores among which $\mathrm{Cy} 3, \mathrm{Cy} 5$ and $\mathrm{Cy} 7$ with increasing $\pi$ conjugation showed gradually red-shifted absorption and emission.
However, this often significantly increases molecular size and hydrophobicity, leading to poor solubility for aqueous-solution based applications such as bioimaging. To red shift the emission of HBT without significantly altering its other physicochemical properties, novel design strategies should be developed.

It is widely known that HBT may emit in the blue light region via its phenolate (enol) form, or in the green-yellow light region via its proton-transferred (keto) form (Figure 1A). The dramatically redshifted emission from the keto form is inspiring for structure optimization. However, this emission happens at little probability only in either the solid state or in aprotic environments. This is because the keto isomer is formed via the mechanism of excited-state intramolecular proton transfer (ESIPT) (Sedgwick et al., 2018), where the formation of an intramolecular hydrogen bonding is required to facilitate the tautomerization to the keto form. In this regard, we envisioned that if we could enhance the chance of intramolecular tautomerization and improve the stability of the keto form, consequently the red-shifted emission can be improved. Since HBT tautomerization necessitates the intramolecular hydrogen bonding between the phenol " $\mathrm{H}$ " and the thiazole "N," we suspected that complexing the phenol "O" and thiazole " $\mathrm{N}$ " with a boron atom may mimic this intramolecular hydrogen bonding effect to improve the chance of tautomerization (Figure 1B). To tune the stability of the tautomerized keto-form isomer, substituents with various electron effects may be incorporated. With these considerations, a series of boron-based HBT derivatives were designed (Figure 1C). In these structures, boroncomplexation was intended to improve the chance of tautomerization; while the substituents meta- or para- to the phenol group was employed to tune the stability of the 
TABLE 1 | Absorption and emission properties of compounds 1-10, 1B-10B in $\mathrm{CH}_{3} \mathrm{CN}$.

\begin{tabular}{|c|c|c|c|c|c|c|}
\hline Compds & $\frac{\lambda_{\mathrm{abs}}}{(\mathrm{nm})}$ & $\frac{\varepsilon}{\left(\mathrm{M}^{-1} \mathrm{~cm}^{-1}\right)}$ & $\frac{\lambda_{\mathrm{em}}}{(\mathrm{nm})}$ & $\frac{\lambda_{\text {ex }}}{(\mathrm{nm})}$ & $\frac{\text { Stokes shift }}{(\mathrm{nm})}$ & $\Phi_{F}$ \\
\hline 1 & 368 & 14,600 & 602 & 374 & 228 & 0.05 \\
\hline $1 B^{a}$ & - & - & - & - & - & - \\
\hline 2 & 327 & 17,300 & 506 & 338 & 168 & 0.04 \\
\hline 2B & 327 & 10,300 & 408 & 358 & 50 & 0.06 \\
\hline 3 & 334 & 22,700 & 442 & 387 & 55 & 0.03 \\
\hline 3B & 334 & 18,400 & 403 & 351 & 52 & 0.27 \\
\hline 4 & 335 & 28,800 & 437 & 388 & 49 & 0.06 \\
\hline 4B & 350 & 9,690 & 407 & 359 & 48 & 0.85 \\
\hline 5 & 353 & 21,800 & 399 & 346 & 53 & 0.10 \\
\hline $5 B$ & 392 & 13,500 & 414 & 391 & 23 & 0.68 \\
\hline 6 & 286 & 23,400 & 499 & 334 & 165 & 0.07 \\
\hline $6 B^{a}$ & - & - & - & - & - & - \\
\hline 7 & 340 & 15,200 & 399 & 342 & 57 & 0.02 \\
\hline $7 B$ & 340 & 17,100 & 502 & 318 & 184 & 0.24 \\
\hline 8 & 342 & 15,300 & 379 & 360 & 19 & 0.03 \\
\hline $8 B$ & 341 & 5,730 & 412 & 361 & 51 & 0.22 \\
\hline 9 & 360 & 13,300 & 401 & 342 & 59 & 0.06 \\
\hline 9B & 360 & 8,560 & 482 & 389 & 93 & 0.26 \\
\hline 10 & 354 & 4,670 & 472 & 354 & 118 & 0.03 \\
\hline 10B & 365 & 1,210 & 597 & 437 & 160 & 0.06 \\
\hline
\end{tabular}

${ }^{a}$ Not detected due to poor solubility.

keto-like resonance structure. Another advantage of this complexation would be to improve the quantum yields of the HBT derivatives by restricting $\mathrm{HBT}$ in a more rigid skeleton and therefore minimizing the excited energy decay. This effect is similar to that shows in BODIPY which also composes a boron-nitrogen complexation unite to lock the structure in a rigid form and consequently make the fluorophore showing robust brightness (Ulrich et al., 2008; Lu et al., 2014). Based on the above considerations, a series of boron-complexed HBT derivatives $\left(\mathbf{H B T}-\mathbf{B F}_{2}\right)$ were designed, with simple substituents of various electron withdrawing or donating effects being incorporated in the meta- or paraposition to the phenol group (Figure 1C). It should be noted that although Santra et al. (2012) reported several of this kind of boron-complexed HBT derivatives, the structurephotophysical property relationship remains underexplored.

These $\mathbf{H B T}-\mathbf{B F}_{\mathbf{2}}$ fluorophores were facilely synthesized by first condensing various substituted salicylic acids with $\mathrm{O}$-aminothiophenol to yield 1-10, followed by complexation with $\mathrm{BF}_{3} \cdot \mathrm{Et}_{2} \mathrm{O}$ to give 1B-10B. Their structures were characterized by ${ }^{1} \mathrm{HNMR},{ }^{13} \mathrm{CNMR}$, ${ }^{19}$ FNMR and MS spectroscopy. Data were detailed in the supporting information.

\section{Photophysical Properties of $\mathrm{HBT}^{-\mathrm{BF}_{2}}$ Derivatives}

After preparation of the compounds, we first tested their photophysical properties, especially their fluorescence emission wavelength and brightness. By measuring their absorption and emission spectra in acetonitrile and ethyl acetate, we observed that $\mathrm{BF}_{2}$-complexation generally caused a ca. $20 \mathrm{~nm}$ red-shift of the HBT absorption, accompanied with decreased absorption intensity (Table 1; $\quad$ Supplementary Figures S1-S4; Supplementary Table S1) and significantly enhanced emission brightness. As shown in Table 1 and Supplementary Table S1, $\mathrm{BF}_{2}$-complexation dramatically enhanced the quantum yields of the HBT compounds. This phenomenon was especially obvious for the compounds bearing the substituents in the meta- position. Taking the data collected in acetonitrile for example, the quantum yield of 4 is 0.06 while that of $\mathbf{4 B}$ is 0.85 , which represented a more than tenfold improvement. This observation agreed with the expectation that improving the rigidity of the structures by $\mathrm{BF}_{2}$ complexation should reduce the excited energy decay to improve their brightness.

When the impact of $\mathrm{BF}_{2}$-complexation on emission maximum was studied, it was found that this should be discussed case-bycase (Figure 2; Supplementary Figures S5, S6). To make it clear, we plotted the maximum emission wavelength of the compounds against the Hammett's constants of the substituents. As shown in Figures 2A,B, for the compounds bearing the substituents in the meta- position without $\mathrm{BF}_{2}$-complxation, their maximum emission wavelength increased as the substituents got more electron-withdrawing (with bigger Hammett's constant); while $\mathrm{BF}_{2}$-complexation generally blue-shifted their maximum emission. For the compounds bearing the substituents in the para- position without $\mathrm{BF}_{2}$-complxation (Figures $2 \mathrm{C}, \mathrm{D}$ ), either the strong electron-withdrawing effect or the electron-donating effect of the substituent resulted in red-shifted emission. $\mathrm{BF}_{2}-$ complexation generally caused a further red-shift effect. Noteworthy, compound $\mathbf{1 0}$ bearing a strong electron-donating amine group in the para- position showed its maximum emission at $472 \mathrm{~nm}$; while $\mathrm{BF}_{2}$-complexation caused a ca $120 \mathrm{~nm}$ red shift, leading to the maximum emission at $597 \mathrm{~nm}$ for $10 \mathrm{~B}$ with part falling into the near-infrared region $(>650 \mathrm{~nm})$. This observation agreed with our ESIPT-mimicking design rationale, as $\mathrm{BF}_{2}$-complexation of the compounds bearing strong electrondonating groups in the para-position should make the structure more easily resonate to the keto-like structure (Figure 1C).

\section{Structure-Property Relationship Guided Design of HBT-BF 2 Derivatives With Near-Infrared Emission}

The above work shed light on the structure-property relationship of the $\mathbf{H B T}-\mathbf{B F}_{2}$ derivatives, and revealed compound $\mathbf{1 0 B}$ as a good starting point for further optimization. To further red-shift the emission of 10B, compounds 11-13 bearing differently sized nitrogen-containing rings were designed and synthesized, together with their $\mathrm{BF}_{2}$-complexed derivatives (Figure $\mathbf{3 A}$ ). The differently sized nitrogen-containing rings were aimed to tune the twisted internal charge transfer effect, a strategy effectively used to improve the emission and brightness of Rhodamine and BODIPY (Grimm et al., 2015; Liu et al., 2016b; Grimm et al., 2017). Compounds 11-13 were synthesized by coupling compound 7 with various amines. Subsequent complexation with $\mathrm{BF}_{3} \cdot \mathrm{Et}_{2} \mathrm{O}$ yielded their $\mathrm{BF}_{2}$-complexed derivatives. Synthetic procedures and structure characterization data were detailed in the supporting information.

Comparison of the absorption and emission spectra of 11-13 with those of 11B-13B showed that $\mathrm{BF}_{2}$ - 

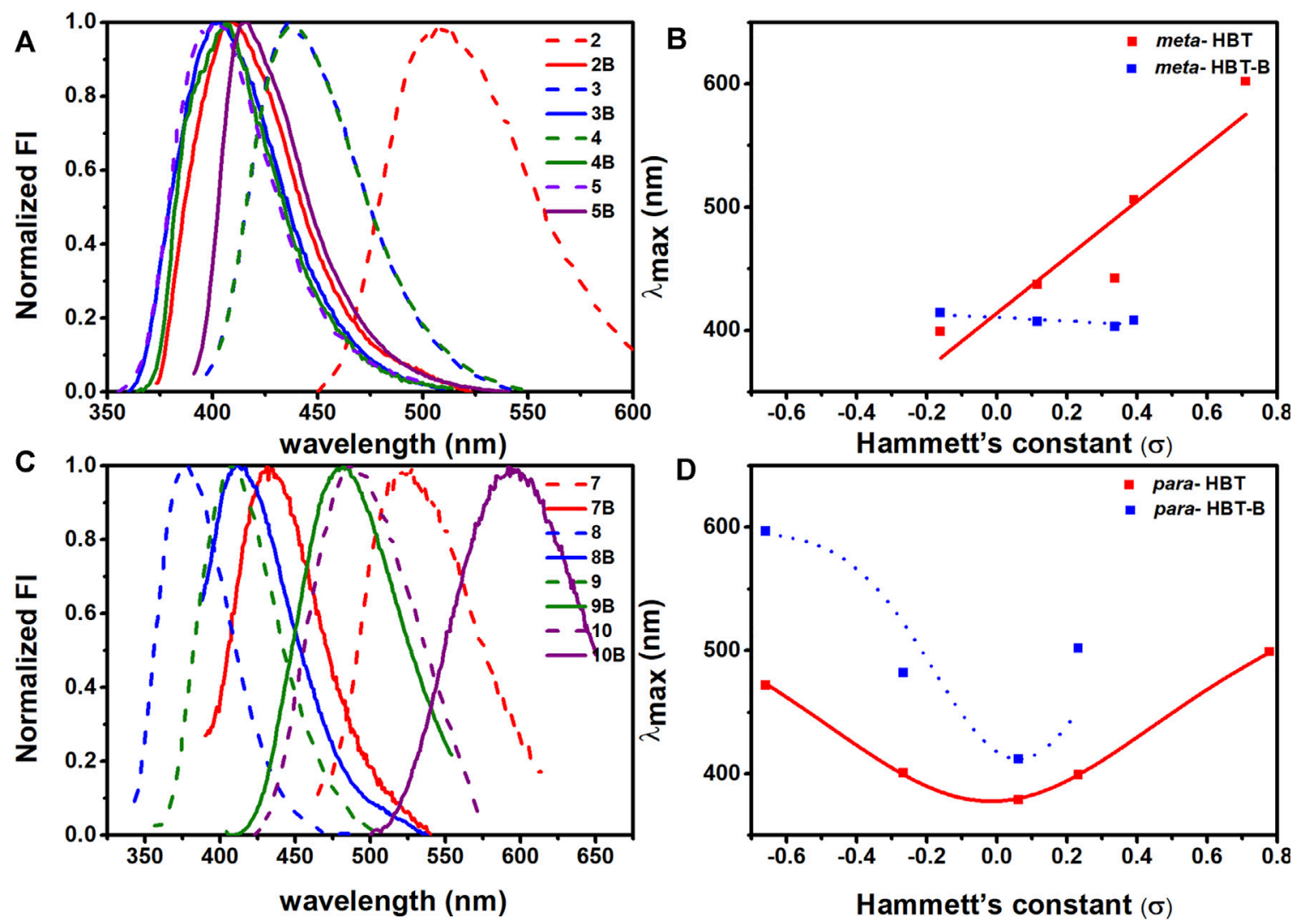

FIGURE 2 | Emission spectra of 2-10, 2B-10B in $\mathrm{CH}_{3} \mathrm{CN}$. (A) Normalized emission spectra of 2-5, 2B-5B in $\mathrm{CH}_{3} \mathrm{CN}$; (B) Plot of the maximum emission wavelength of 2-5, 2B-5B against the Hammet's constant of the R substituent. (C) Normalized emission spectra of $\mathbf{7 - 1 0}, \mathbf{7 B - 1 0 B}$ in $\mathrm{CH}_{3} \mathrm{CN}$; (D) Plot of the maximum emission wavelength of $\mathbf{7 - 1 0}, \mathbf{7 B - 1 0 B}$ against the Hammett's constant of the R substituent.
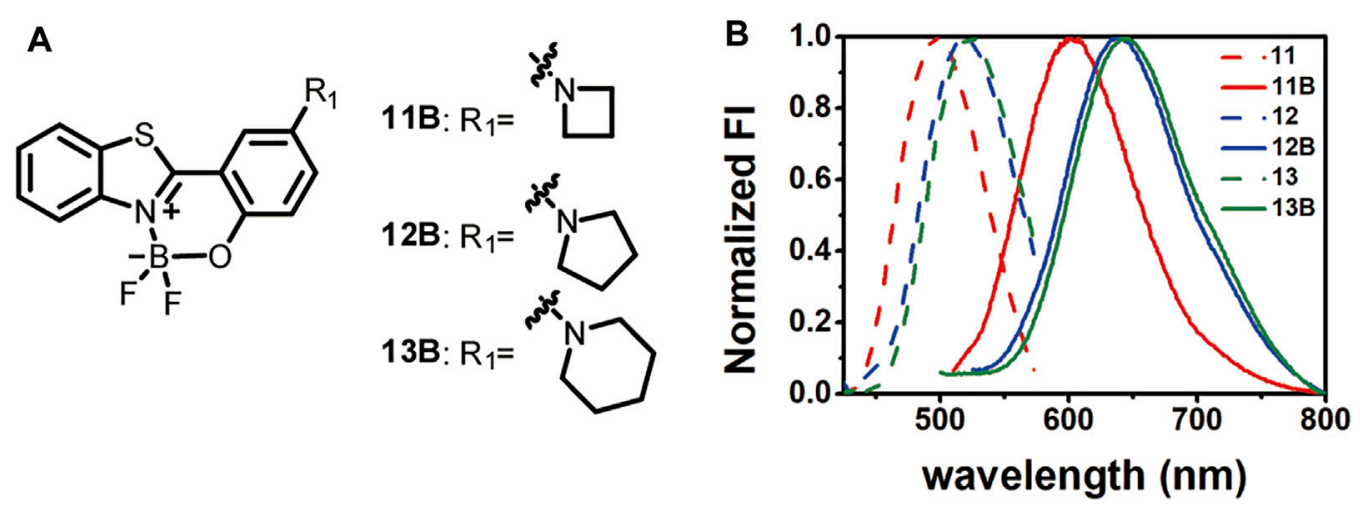

FIGURE 3 | Structures of 11B-13B (A) and their emission spectra in comparison to those before BF ${ }_{2}$-complexation in acetonitrile (B).

complexation resulted in a dramatic red-shifting effect, accompanied by the dramatic improvement of the fluorescence quantum yields (Figure 3B; Table 2; Supplementary Figures S7-S9). Noteworthy, 12B and 13B bearing a pyrrolidone and a piperidine ring, respectively, showed their emission bands centred around $650 \mathrm{~nm}$ in acetonitrile. Given their small size, they are among the smallest organic molecules showing NIR emission.

To further understand the relationship between the fluorescence properties and the chemical structures of these boron-based HBT derivatives, we first measured the IR spectra of 10-13 and 10B-13B (Table 3). It was observed the $\mathrm{BF}_{2}$ 
TABLE 2 | Optical properties of compounds 11-13, 11B-13B

\begin{tabular}{|c|c|c|c|c|c|c|}
\hline Comp & $\frac{\lambda_{\text {abs }}}{(n m)}$ & $\frac{\varepsilon}{\left(\mathrm{M}^{-1} \mathrm{~cm}^{-1}\right)}$ & $\frac{\lambda_{\mathrm{em}}}{(\mathrm{nm})}$ & $\frac{\lambda_{\text {ex }}}{(n m)}$ & $\frac{\text { Stokes shift }}{(\mathrm{nm})}$ & $\Phi_{\mathrm{F}}$ \\
\hline \multirow[t]{2}{*}{11} & 404 & 5,980 & 470 & 377 & 93 & $0.02^{a}$ \\
\hline & 400 & 5,940 & 496 & 375 & 121 & $0.01^{b}$ \\
\hline \multirow[t]{2}{*}{12} & 422 & 5,960 & 482 & 391 & 91 & $0.02^{a}$ \\
\hline & 422 & 5,670 & 517 & 396 & 121 & $0.01^{b}$ \\
\hline \multirow[t]{2}{*}{13} & 383 & 7,100 & 494 & 357 & 137 & $0.02^{a}$ \\
\hline & 383 & 6,660 & 523 & 357 & 166 & $0.01^{b}$ \\
\hline \multirow[t]{2}{*}{ 11B } & 404 & 4,530 & 586 & 457 & 129 & $0.58^{a}$ \\
\hline & 403 & 4,120 & 601 & 447 & 154 & $0.16^{b}$ \\
\hline \multirow[t]{2}{*}{$12 B$} & 423 & 6,180 & 616 & 482 & 134 & $0.93^{a}$ \\
\hline & 426 & 5,110 & 641 & 482 & 159 & $0.22^{b}$ \\
\hline \multirow[t]{2}{*}{$13 B$} & 383 & 3,730 & 618 & 430 & 188 & $0.46^{a}$ \\
\hline & 385 & 3,490 & 646 & 421 & 225 & $0.10^{b}$ \\
\hline
\end{tabular}

${ }^{a}$ Data measured in EtOAC.

${ }^{b}$ Data measured in $\mathrm{CH}_{3} \mathrm{CN}$.

complexation generally shifted the $v_{\mathrm{C}=\mathrm{N}}$ to higher wavenumbers, and the $v_{(\mathrm{ph}) \mathrm{C}-\mathrm{N}}$ was also shifted a little to higher wavenumbers (Supplementary Figure S10). These results suggested that $\mathrm{BF}_{2}$ complexation enhanced both the electron withdrawing effect of the benzothiazole moiety and the electron donating effect of the amino substituent para to the phenol group when the compounds were in their ground state. Density functional theory (DFT) and time dependent density functional theory (TDDFT) calculations were also carried out with the B3LYP/6$31+\mathrm{G}(\mathrm{d})$ method basis set using the Gaussian 09 C.01 program to further understand the structure-property relationship. The optimized geometry, the highest occupied molecular orbital (HOMO) and the lowest unoccupied molecular orbital (LUMO) of these compounds are presented in Figure 4. The results showed that complexing the heteroatoms in 10-13 with boron to lock the structures in a more rigid form (10B-13B) decreased their HOMO-LUMO energy gaps, which may explain their red-shifted emissions. Generally, a negative correlation can be noticed between the emission wavelength and the HOMO-LUMO energy gap for 10B-13B, with 10B showing the biggest energy gap demonstrating the shortest emission wavelength. Noteworthy, 12B and 13B demonstrated similar HOMO-LUMO energy gap values, which was in consistence with their similar emission spectra.

\section{Applicability of 13B for Cell Imaging}

Finally, taking compounds 13 and 13B as representatives, we tested the feasibility of the compounds for cell imaging. For confocal fluorescence imaging, HeLa cells were first stained with $20 \mu \mathrm{M}$ compounds 13 or $13 \mathrm{~B}$ at $37^{\circ} \mathrm{C}$ for $30 \mathrm{~min}$. After that, the cells were washed three times with PBS and then imaged. As shown in Figure 5, both compounds 13 and 13B demonstrate good cell membrane permeability and showed bright green and red light in $\mathrm{HeLa}$ cells, respectively. Obviously, compared with 13, 13B with a near infrared window emission, owned more widely applications in the fields of bioimaging as well as others.

\section{DISCUSSION}

Mimicking the ESIPT effect, we have developed a series of new HBT-based fluorophores by complexing the two key heteroatoms in HBT with a boron atom. These derivatives showed dramatically improved quantum yields. We also carried out a simple structure-property relationship study which showed that electron-donating substituents in the para-position to the phenol group in HBT should dramatically red-shift the emission of the HBT-B derivatives. Based on this observation, we developed compounds $\mathbf{1 2 B}$ and $\mathbf{1 3 B}$ whose relative molecular masses were below 350 but demonstrated nearinfrared emission in acetonitrile, which made them among the smallest structures demonstrating near-infrared emission. These results highlighted the advantage of employing naturally occurring phenomena to design new materials with improved properties.

\section{MATERIALS AND METHODS}

\section{Materials and Instruments for Synthesis}

All reagents for synthesis were purchased at analytical grade and used without further purification except otherwise noted. Thin-layer chromatography (TLC, $0.25 \mathrm{~mm}$ silica gel plates, 60 F-254, Merker KGaA) was employed to monitor the reactions and UV light was used as the visualizing agent. Flash column chromatrography over silica gel $(40-63 \mu \mathrm{m}$ particle size, Taiyang, Rushan) was employed for compound purification. ${ }^{1} \mathrm{H}$ NMR and ${ }^{13} \mathrm{C}$ NMR spectra were recorded on a Bruker spectrometer (500 and $126 \mathrm{MHz}$, respectively) at $23^{\circ} \mathrm{C}$. NMR spectra were calibrated using TMS as the internal references. All chemical shifts were reported in parts per million (ppm) and coupling constants $(J)$ in $\mathrm{Hz}$. Mass spectra (HRMS) were measured on an Agilent 6224 TOF LC/MS spectrometer or a Shimadzu LCMS-2020 mass spectrometer.

\section{General Procedures for the Synthesis of Compounds 1-10, 1B-10B}

A mixture of $o$-aminothiophenol, the salicylic acid derivative bearing the desired substituent, tetrabutylammonium bromide, and

TABLE 3 | Typical IR data (in $\mathrm{cm}^{-1}$ ) for compounds 11-13, 11B-13B

\begin{tabular}{lcccrrrr}
\hline Bond & $\mathbf{1 0}$ & $\mathbf{1 0 B}$ & $\mathbf{1 1}$ & $\mathbf{1 1 B}$ & $\mathbf{1 2}$ & $\mathbf{1 2 B}$ & $\mathbf{1 3}$ \\
\hline$v_{\mathrm{C}=\mathrm{N}}$ & $1,499.38$ & $1,504.1$ & $1,493.6$ & $1,522.2$ & $1,504.2$ & $1,520.6$ \\
$v_{(\mathrm{ph}) \mathrm{C}-\mathrm{N}}$ & $1,441.5$ & $1,466.5$ & $1,426.1$ & $1,478.1$ & $1,431.9$ & $1,409.9$
\end{tabular}


LUMO:
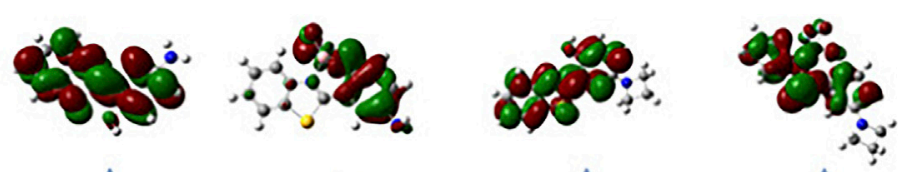

$0.13269 \hat{\mathrm{eV}}$
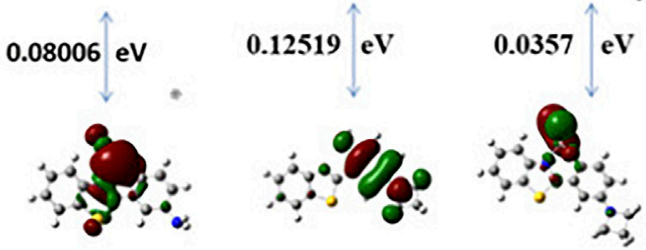

HOMO:
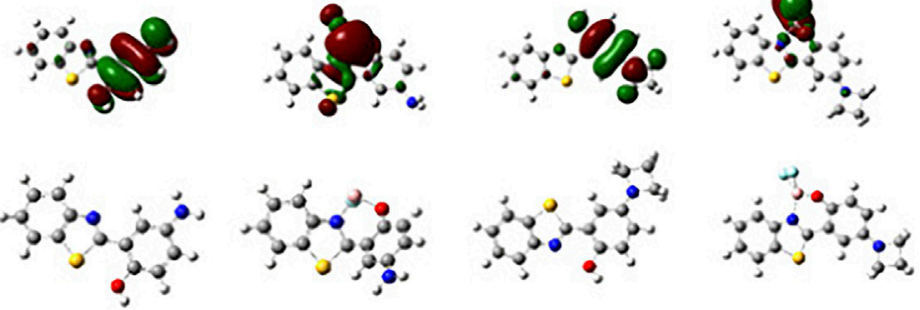

10

10B

11

11B

LUMO:
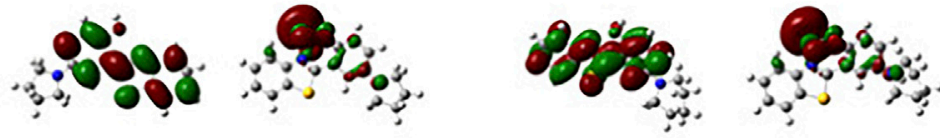

$0.12414 \hat{\mathrm{eV}} \quad 0.05621 \mathrm{eV}$

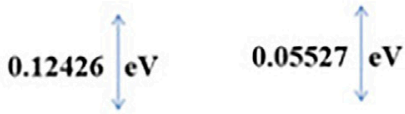

HOMO:
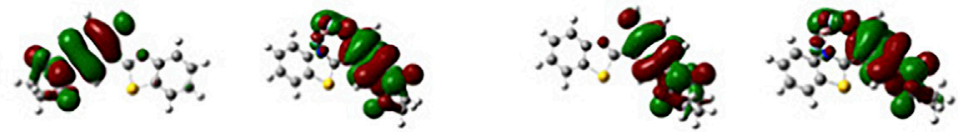

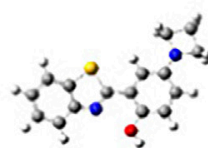

12

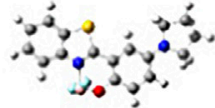

12B

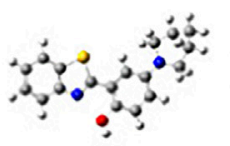

13

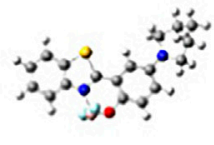

13B

FIGURE 4 |DFT optimized structures and molecular orbital plots (LUMO and HOMO) of selected fluorophores based on the optimized ground-state geometry ( $\mathrm{S}_{0}$ ). In the ball-and-stick representation, carbon, nitrogen, and oxygen atoms are colored gray, blue, and red, respectively.

triphenyl phosphite was heated at $120^{\circ} \mathrm{C}$ for $2 \mathrm{~h}$. After cooling down to ambient temperature, $\mathrm{MeOH}$ was added to the mixture and the mixture was sonicated. The solid was isolated by filtration and washed with $\mathrm{MeOH}$, which was further purified by recrystallization to give the $\mathrm{HBT}$ derivative (1-10) ready for $\mathrm{BF}_{2}$-complexation.

For $\mathrm{BF}_{2}$-complexation, each one of compounds 1-10 was dissolved in dry dichloromethane, and was treated with triethylamine. $\mathrm{BF}_{3} \cdot \mathrm{Et}_{2} \mathrm{O}$ was added dropwise to the solution at room temperature under nitrogen atmosphere. The solution was stirred at room temperature for $1 \mathrm{~h}$. After the disappearance of the starting material as monitored by TLC, the solvent was directly evaporated under reduced pressure. The crude product was purified by silica gel column chromatography (eluent: PE and EA). Structure characterization data can be found in the supporting information.

\section{Synthesis for Compounds 11-13, 11B-13B}

These compounds were synthesized starting with compound 7. Compound 7 was first protected of the phenol group; then underwent coupling reaction to install the amino substitutent; subsequent deprotection yielded 10-13; and final $\mathrm{BF}_{2}$ complexation gave 11B-13B.

In detail, to a solution of compound $7(1.0 \mathrm{mmol})$ and potassium carbonate $(2.0 \mathrm{mmol})$ in dry DMF $(5.0 \mathrm{ml})$ was added $\mathrm{MOMCl}(3 \mathrm{mmol})$ dropwise at $0^{\circ} \mathrm{C}$. The resulting mixture was stirred at room temperature for $3 \mathrm{~h}$. After the reaction was compete as monitored by TLC, the mixture was extracted with ethyl acetate. The obtained organic layer was successively washed with distilled water and brine, and was subsequently dried over anhydrous sodium sulphate. After removal of the solvent, the desired product was obtained which was used without further purification. 

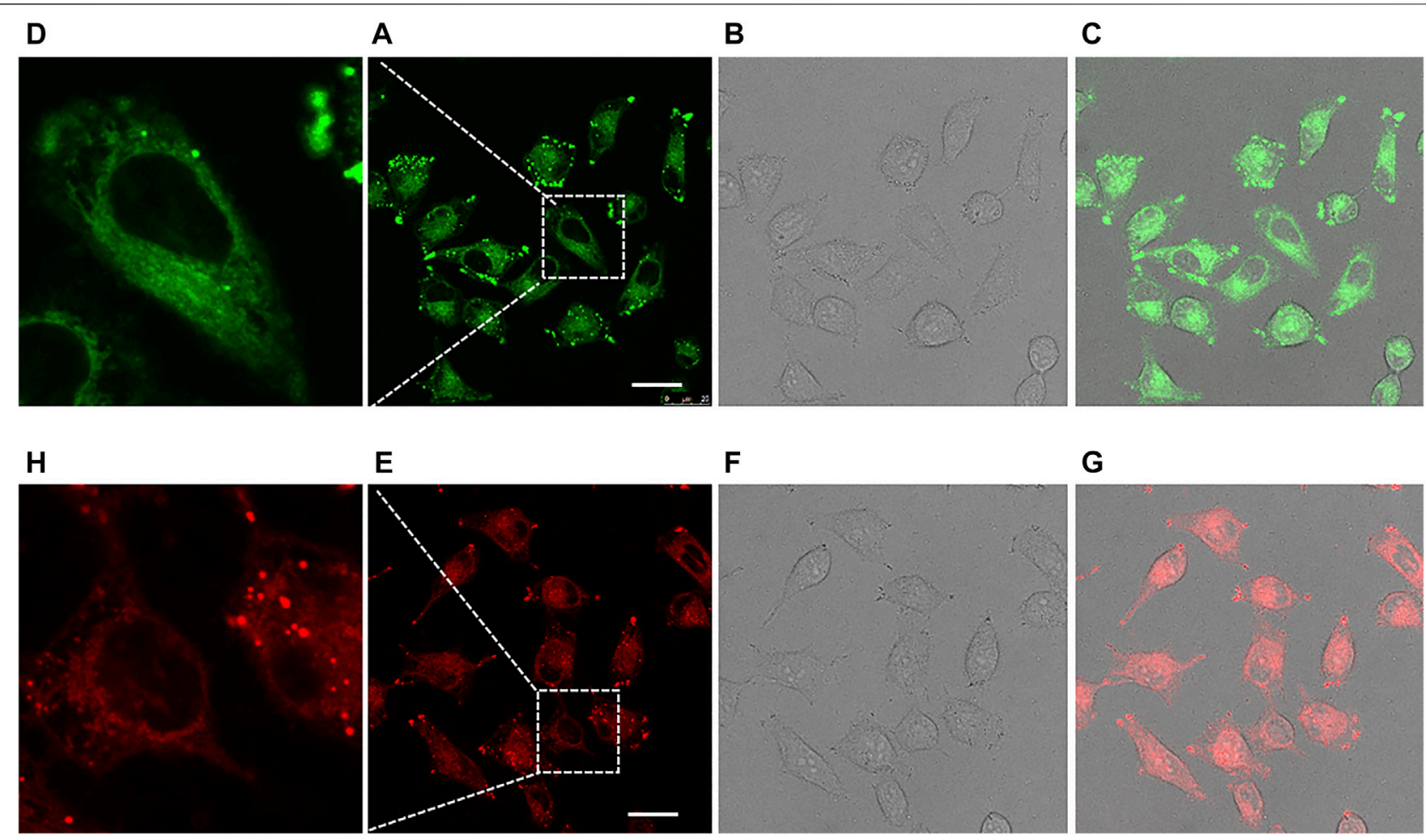

\section{E}

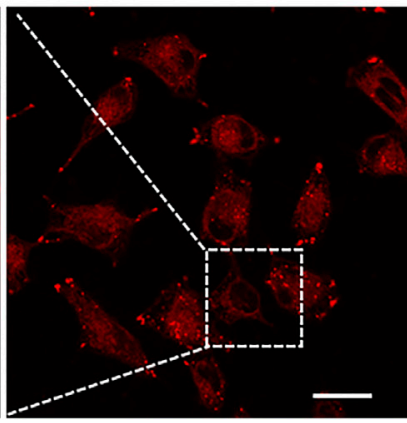

$\mathbf{F}$

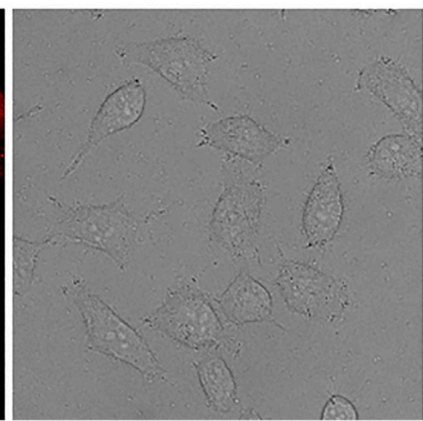

G

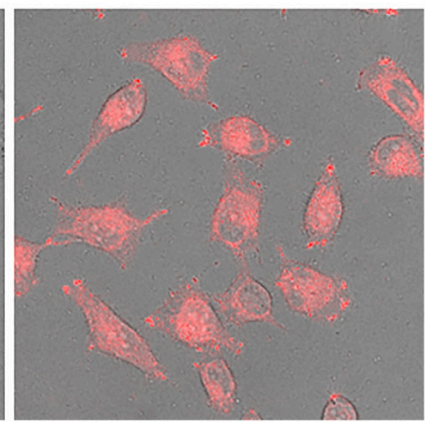

FIGURE 5| Confocal fluorescence imaging of HeLa cells cultured with 13 [Ex: 405 nm; Em: 480-600 nm; (A-D)] and 13B [Ex: 405 nm; Em: $550-700$ nm; (E-H)] for $30 \mathrm{~min}$. Each compound was administrated at $20 \mu \mathrm{M}$. The 2 nd row of pictures represented the images taken under fluorescence field, with partially expanded view shown in the 1 st row. The 3rd row of pictures represented the images taken under bright field. The 4 th row of pictures were merged ones. Scale bar: $25 \mu \mathrm{M}$.

${ }^{1} \mathrm{H}$ NMR $\left(500 \mathrm{MHz}, \mathrm{CDCl}_{3}\right) \delta 8.69(\mathrm{~d}, J=2.5 \mathrm{~Hz}, 1 \mathrm{H}), 8.10(\mathrm{~d}$, $J=8.1 \mathrm{~Hz}, 1 \mathrm{H}), 7.92(\mathrm{~d}, J=7.9 \mathrm{~Hz}, 1 \mathrm{H}), 7.51(\mathrm{ddd}, J=9.8,7.9$, $1.9 \mathrm{~Hz}, 2 \mathrm{H}), 7.42-7.36(\mathrm{~m}, 1 \mathrm{H}), 7.16(\mathrm{~d}, J=8.9 \mathrm{~Hz}, 1 \mathrm{H}), 5.40$ (s, 2H), $3.56(\mathrm{~s}, 3 \mathrm{H}) \cdot{ }^{13} \mathrm{C} \mathrm{NMR}\left(125 \mathrm{MHz}, \mathrm{CDCl}_{3}\right) \delta 161.31$, $153.74,151.98,136.15,134.12,131.89,126.18,125.04,124.59$, $123.07,121.21,116.47,114.89,94.55,56.73$.

To a solution of MOM protected $7(1.0 \mathrm{mmol})$ and the corresponding amine $(2.0 \mathrm{mmol})$ in dry toluene $(5.0 \mathrm{ml})$ was added cesium carbonate $(3 \mathrm{mmol})$ as a base, and subsequently added $\operatorname{Pd}_{2}(\mathrm{dba})_{3}$ and BINAP. Under a nitrogen atmosphere, the mixture was refluxed overnight. After the reaction was completed as monitored by TLC, the reaction mixture was filtered through Celite and the filtrate was evaporated under reduced pressure. The crude product was purified by silica gel column chromatography to yield the MOM protected 11-13 (eluent: $\mathrm{PE}$ and EA). These compounds were then individually dissolved in $\mathrm{THF} / \mathrm{H}_{2} \mathrm{O}$, treated with $\mathrm{HCl}$ $(5 \mathrm{~N})$ at $0^{\circ} \mathrm{C}$. The mixture was stirred at $50^{\circ} \mathrm{C}$ overnight. After evaporation of the volatile solvent, the mixture was diluted with water and extracted with ethyl acetate. The organic phase was washed with brine, dried over anhydrous $\mathrm{Na}_{2} \mathrm{SO}_{4}$, and evaporated under reduced pressure. The residue was purified by silica gel column chromatography to give 11-13 (eluent: $\mathrm{PE}$ and $\mathrm{EA}) . \mathrm{BF}_{2^{-}}$ complexation was carried out as afore mentioned to give 11B-13B. Structure characterization data can be found in the supporting information.

\section{General Experimental for Photophysical Property Characterization}

All the photophysical characterization experiments were carried out at ambient temperature. Fluorescence measurements were acquired using an Agilent Cary Eclipse Fluorescence spectrophotometer. Absorption spectra were performed on a U-3010 Spectrophotometer.

\section{Quantum Yields Determination}

Quantum yields were determined using quinine sulfate $\left(\Phi_{\text {standard }}=0.577\right.$ in $\left.0.1 \mathrm{M} \mathrm{H}_{2} \mathrm{SO}_{4}\right)$ as astandard according to a published method. The quantum yield was calculated according to the equation:

$$
\Phi_{\text {sample }}=\Phi_{\text {stan dard }} \cdot \frac{\mathrm{Abs}_{\text {stan dard }} \cdot \sum \mathrm{F}_{\text {sample }}}{\mathrm{Abs}_{\text {sample }} \cdot \sum \mathrm{F}_{\text {stan dard }}} \cdot \frac{n_{\text {sample }^{2}}}{n_{\text {stan dard }}{ }^{2}}
$$

where $\Phi$ is the quantum yield, $\Sigma_{\mathrm{F}}$ is the integrated fluorescence intensity, Abs is absorbance at $\lambda_{e x} 375 \mathrm{~nm}$, and $n$ represents the refractive index of the solvent. 


\section{Cell Imaging}

Hela cells were cultured on glass-bottom 6-well plates overnight, then incubated with 13 or $13 \mathrm{~B}(20 \mu \mathrm{M})$ at $37^{\circ} \mathrm{C}$ for $30 \mathrm{~min}$. After three quick wash with PBS, cells were observed under a confocal microscopy (For 13, excitation was conducted at $405 \mathrm{~nm}$ and emission was collected at 480-600 nm. For 13B, excitation was conducted at $405 \mathrm{~nm}$ and emission was collected at $550-700 \mathrm{~nm}$ ).

\section{DATA AVAILABILITY STATEMENT}

The original contributions presented in the study are included in the article/Supplementary Material, further inquiries can be directed to the corresponding author.

\section{AUTHOR CONTRIBUTIONS}

$\mathrm{XL}$ designed the project. YR and LZ synthesized the compounds. YR measured the photophysical properties of the compounds, and performed the imaging experiments. XL

\section{REFERENCES}

Barman, S., Mukhopadhyay, S. K., Biswas, S., Nandi, S., Gangopadhyay, M., Dey, S., et al. (2016). A p -Hydroxyphenacyl-Benzothiazole-Chlorambucil Conjugate as a Real-Time-Monitoring Drug-Delivery System Assisted by Excited-State Intramolecular Proton Transfer. Angew. Chem. Int. Ed. 55 (13), 4194-4198. doi:10.1002/anie.201508901

Boens, N., Leen, V., and Dehaen, W. (2012). Fluorescent Indicators Based on BODIPY. Chem. Soc. Rev. 41 (3), 1130-1172. doi:10.1039/ clcs $15132 \mathrm{k}$

Cheng, Y., Li, G., Liu, Y., Shi, Y., Gao, G., Wu, D., et al. (2016). Unparalleled Ease of Access to a Library of Biheteroaryl Fluorophores via Oxidative Cross-Coupling Reactions: Discovery of Photostable NIR Probe for Mitochondria. J. Am. Chem. Soc. 138 (14), 4730-4738. doi:10.1021/jacs.5b09241

Chu, Y.-H., Escobedo, J. O., Jiang, M., Steyger, P. S., and Strongin, R. M. (2016). Rhodamine Analogs for Molecular Ruler Applications. Dyes Pigm. 126, 46-53. doi:10.1016/j.dyepig.2015.11.015

Fernández, A., and Vendrell, M. (2016). Smart Fluorescent Probes for Imaging Macrophage Activity. Chem. Soc. Rev. 45, 1182-1196. doi:10.1039/ c5cs00567a

Fu, M., Xiao, Y., Qian, X., Zhao, D., and Xu, Y. (2008). A Design Concept of LongWavelength Fluorescent Analogs of Rhodamine Dyes: Replacement of Oxygen with Silicon Atom. Chem. Commun. 15 (15), 1780-1782. doi:10.1039/b718544h

Goryaynov, S. A., Okhlopkov, V. A., Golbin, D. A., Chernyshov, K. A., Svistov, D. V., Martynov, B. V., et al. (2019). Fluorescence Diagnosis in Neurooncology: Retrospective Analysis of 653 Cases. Front. Oncol. 9, 830. doi:10.3389/ fonc. 2019.00830

Grimm, J. B., English, B. P., Chen, J., Slaughter, J. P., Zhang, Z., Revyakin, A., et al. (2015). A General Method to Improve Fluorophores for Live-Cell and SingleMolecule Microscopy. Nat. Methods 12 (3), 244-250. doi:10.1038/nmeth.3256

Grimm, J. B., Muthusamy, A. K., Liang, Y., Brown, T. A., Lemon, W. C., Patel, R., et al. (2017). A General Method to Fine-Tune Fluorophores for Live-Cell and In Vivo Imaging. Nat. Methods 14 (10), 987-994. doi:10.1038/nmeth.4403

Grimm, J. B., Tkachuk, A. N., Xie, L., Choi, H., Mohar, B., Falco, N., et al. (2020). A General Method to Optimize and Functionalize Red-Shifted Rhodamine Dyes. Nat. Methods 17 (8), 815-821. doi:10.1038/s41592-020-0909-6

Hernot, S., van Manen, L., Debie, P., Mieog, J. S. D., and Vahrmeijer, A. L. (2019). Latest Developments in Molecular Tracers for Fluorescence Image-Guided and YR drafted the manuscript. All authors read and agreed the manuscript.

\section{FUNDING}

This work was supported by the National Natural Science Foundations of China (21778048), and the Key Research and Development Program of Zhejiang Province (2021C03045).

\section{ACKNOWLEDGMENTS}

We appreciate Prof Jianzhong Chen for the help in conducting the TDDFT calculations.

\section{SUPPLEMENTARY MATERIAL}

The Supplementary Material for this article can be found online at: https:/www.frontiersin.org/articles/10.3389/fchem.2021.807433/ full\#supplementary-material

Cancer Surgery. Lancet Oncol. 20 (7), e354-e367. doi:10.1016/s1470-2045(19) 30317-1

Kim, H. M., and Cho, B. R. (2015). Small-molecule Two-Photon Probes for Bioimaging Applications. Chem. Rev. 115 (11), 5014-5055. doi:10.1021/ cr5004425

Koide, Y., Urano, Y., Hanaoka, K., Piao, W., Kusakabe, M., Saito, N., et al. (2012). Development of NIR Fluorescent Dyes Based on Si-Rhodamine for In Vivo Imaging. J. Am. Chem. Soc. 134 (11), 5029-5031. doi:10.1021/ ja210375e

Lefevre, C., Kang, H. C., Haugland, R. P., Malekzadeh, N., Arttamangkul, S., and Haugland, R. P. (1996). Texas Red-X and Rhodamine Red-X, New Derivatives of Sulforhodamine 101 and Lissamine Rhodamine B with Improved Labeling and Fluorescence Properties. Bioconjug. Chem. 7 (4), 482-489. doi:10.1021/ bc960034p

Lei, Z., Li, X., Luo, X., He, H., Zheng, J., Qian, X., et al. (2017). Bright, Stable, and Biocompatible Organic Fluorophores Absorbing/Emitting in the Deep NearInfrared Spectral Region. Angew. Chem. Int. Ed. 56 (11), 2979-2983. doi:10.1002/anie.201612301

Li, D. H., Schreiber, C. L., and Smith, B. D. (2020a). Sterically Shielded Heptamethine Cyanine Dyes for Bioconjugation and High Performance Near-Infrared Fluorescence Imaging. Angew. Chem. Int. Ed. 59 (29), 12154-12161. doi:10.1002/anie.202004449

Li, X., Qiu, W., Li, J., Chen, X., Hu, Y., Gao, Y., et al. (2020b). First-generation Species-Selective Chemical Probes for Fluorescence Imaging of Human Senescence-Associated $\beta$-galactosidase. Chem. Sci. 11 (28), 7292-7301. doi:10.1039/d0sc01234c

Li, X., Tao, R.-R., Hong, L.-J., Cheng, J., Jiang, Q., Lu, Y.-M., et al. (2015). Visualizing Peroxynitrite Fluxes in Endothelial Cells Reveals the Dynamic Progression of Brain Vascular Injury. J. Am. Chem. Soc. 137 (38), 12296-12303. doi: $10.1021 /$ jacs.5b06865

Liu, J., Sun, Y.-Q., Zhang, H., Shi, H., Shi, Y., and Guo, W. (2016a). SulfoneRhodamines: A New Class of Near-Infrared Fluorescent Dyes for Bioimaging. ACS Appl. Mater. Inter. 8 (35), 22953-22962. doi:10.1021/ acsami.6b08338

Liu, X., Qiao, Q., Tian, W., Liu, W., Chen, J., Lang, M. J., et al. (2016b). Aziridinyl Fluorophores Demonstrate Bright Fluorescence and superior Photostability by Effectively Inhibiting Twisted Intramolecular Charge Transfer. J. Am. Chem. Soc. 138 (22), 6960-6963. doi:10.1021/ jacs.6b03924 
Liu, Y., Li, C., Ren, Z., Yan, S., and Bryce, M. R. (2018). All-Organic Thermally Activated Delayed Fluorescence Materials for Organic Light-Emitting Diodes. Nat. Rev. Mater. 3 (4), 1-20. doi:10.1038/natrevmats.2018.20

Lu, H., Mack, J., Yang, Y., and Shen, Z. (2014). Structural Modification Strategies for the Rational Design of Red/NIR Region BODIPYs. Chem. Soc. Rev. 43 (13), 4778-4823. doi:10.1039/c4cs00030g

Michie, M. S., Götz, R., Franke, C., Bowler, M., Kumari, N., Magidson, V., et al. (2017). Cyanine Conformational Restraint in the Far-Red Range. J. Am. Chem. Soc. 139 (36), 12406-12409. doi:10.1021/jacs.7b07272

Qian, H., Cousins, M. E., Horak, E. H., Wakefield, A., Liptak, M. D., and Aprahamian, I. (2017). Suppression of Kasha's Rule as a Mechanism for Fluorescent Molecular Rotors and Aggregation-Induced Emission. Nat. Chem. 9 (1), 83-87. doi:10.1038/nchem.2612

Santra, M., Moon, H., Park, M.-H., Lee, T.-W., Kim, Y. K., and Ahn, K. H. (2012). Dramatic Substituent Effects on the Photoluminescence of boron Complexes of 2-(benzothiazol-2-yl)phenols. Chem. Eur. J. 18 (32), 9886-9893. doi:10.1002/chem.201200726

Sedgwick, A. C., Wu, L., Han, H.-H., Bull, S. D., He, X.-P., James, T. D., et al. (2018). Excited-State Intramolecular Proton-Transfer (ESIPT) Based Fluorescence Sensors and Imaging Agents. Chem. Soc. Rev. 47 (23), 8842-8880. doi:10.1039/c8cs00185e

Singh, H., Tiwari, K., Tiwari, R., Pramanik, S. K., and Das, A. (2019). Small Molecule as Fluorescent Probes for Monitoring Intracellular Enzymatic Transformations. Chem. Rev. 119 (22), 11718-11760. doi:10.1021/ acs.chemrev.9b00379

Song, X., Johnson, A., and Foley, J. (2008). 7-Azabicyclo[2.2.1]heptane as a Unique and Effective Dialkylamino Auxochrome Moiety: Demonstration in a Fluorescent Rhodamine Dye. J. Am. Chem. Soc. 130 (52), 17652-17653. doi:10.1021/ja8075617

Tian, X., Murfin, L. C., Wu, L., Lewis, S. E., and James, T. D. (2021). Fluorescent Small Organic Probes for Biosensing. Chem. Sci. 12 (10), 3406-3426. doi:10.1039/d0sc06928k

Ulrich, G., Ziessel, R., and Harriman, A. (2008). The Chemistry of Fluorescent Bodipy Dyes: Versatility Unsurpassed. Angew. Chem. Int. Ed. 47 (7), 1184-1201. doi:10.1002/anie.200702070

Usama, S. M., Thompson, T., and Burgess, K. (2019). Productive Manipulation of Cyanine Dye $\pi$-Networks. Angew. Chem. Int. Ed. 58 (27), 8974-8976. doi:10.1002/anie.201902956
Vahrmeijer, A. L., Hutteman, M., Van Der Vorst, J. R., Van De Velde, C. J. H., and Frangioni, J. V. (2013). Image-guided Cancer Surgery Using Near-Infrared Fluorescence. Nat. Rev. Clin. Oncol. 10 (9), 507-518. doi:10.1038/nrclinonc.2013.123

Wang, J., Boens, N., Jiao, L., and Hao, E. (2020). Aromatic [b]-Fused BODIPY Dyes as Promising Near-Infrared Dyes. Org. Biomol. Chem. 18 (22), 4135-4156. doi:10.1039/d0ob00790k

Xiang, Z., Wang, Z.-Y., Ren, T.-B., Xu, W., Liu, Y.-P., Zhang, X.-X., et al. (2019). A General Strategy for Development of a Single Benzene Fluorophore with Full-Color-Tunable, Environmentally Insensitive, and Two-Photon Solid-State Emission. Chem. Commun. 55 (76), 11462-11465. doi:10.1039/c9cc06260b

Yang, Z., Mao, Z., Xie, Z., Zhang, Y., Liu, S., Zhao, J., et al. (2017). Recent Advances in Organic Thermally Activated Delayed Fluorescence Materials. Chem. Soc. Rev. 46 (3), 915-1016. doi:10.1039/c6cs00368k

Yuan, L., Lin, W., Yang, Y., and Chen, H. (2012). A Unique Class of NearInfrared Functional Fluorescent Dyes with Carboxylic-Acid-Modulated Fluorescence ON/OFF Switching: Rational Design, Synthesis, Optical Properties, Theoretical Calculations, and Applications for Fluorescence Imaging in Living Animals. J. Am. Chem. Soc. 134 (2), 1200-1211. doi:10.1021/ja209292b

Conflict of Interest: The authors declare that the research was conducted in the absence of any commercial or financial relationships that could be construed as a potential conflict of interest.

Publisher's Note: All claims expressed in this article are solely those of the authors and do not necessarily represent those of their affiliated organizations, or those of the publisher, the editors and the reviewers. Any product that may be evaluated in this article, or claim that may be made by its manufacturer, is not guaranteed or endorsed by the publisher.

Copyright $(2021$ Ren, Zhou and Li. This is an open-access article distributed under the terms of the Creative Commons Attribution License (CC BY). The use, distribution or reproduction in other forums is permitted, provided the original author(s) and the copyright owner(s) are credited and that the original publication in this journal is cited, in accordance with accepted academic practice. No use, distribution or reproduction is permitted which does not comply with these terms. 